Hagen Lesch ${ }^{*}$

\title{
Konflikteskalation in Tarifverhandlungen: Methode, Indikatoren und empirische Befunde ${ }^{* *}$
}

\section{Zusammenfassung}

Es ist üblich, die Stabilität der Arbeitsbeziehungen anhand verschiedener Arbeitskampfindikatoren zu messen. Da diese aber nur an „materiellen“ Konflikthandlungen wie Streiks und Aussperrungen anknüpfen, wird ein Konfliktmaß entwickelt, das nicht nur materielle Konflikthandlungen berücksichtigt, sondern auch verbale und formale Eskalationsstufen wie Streikdrohungen, Verhandlungsabbrïche oder Schlichtungen. Diese Konflikthandlungen werden in einer Eskalationspyramide so angeordnet, dass sie die Zuspitzung von Tarifkonflikten abbilden. Diese Methode liefert eine Grundlage, um in einem weiteren Schritt zwei Eskalationsmaße abzuleiten: Die maximale Eskalationsstufe gibt als statisches Maß an, bis zu welcher Stufe ein Konflikt eskaliert, die Konfliktintensität summiert als dynamisches Maß alle Konflikthandlungen. Eine Auswertung von 144 Tarifkonflikten in 13 Branchen zeigt bei beiden Maßen große Unterschiede. Außerdem zeigen vier abgeleitete Konfliktlösungsmuster, dass die Tarifverhandlungen in Deutschland nach wie vor als „Konfliktpartnerschaft“ beschrieben werden können. Dabei lassen sich vor allem verbal-formale Konfliktlösungsansätze und materielle Konfliktlösungsansätze unterscheiden.

Key words: Tarifverhandlungen, Konfliktintensität, Arbeitskampf, Sozialpartnerschaft (JEL 51, $52,53)$

\section{Escalation of collective bargaining: Methods, indicators and empirical findings}

\section{Abstract}

Usually, the stability of labour relations is evaluated by a set of different strike indicators. However, these indicators are often only linked to "material" conflict actions like strike and lockouts. Therefore,

* Dr. Hagen Lesch, Jg. 1964, Institut der deutschen Wirtschaft Köln, Leiter Kompetenzfeld Tarifpolitik und Arbeitsbeziehungen, Konrad-Adenauer-Ufer 21, D - 50668 Köln.

E-Mail: lesch@iwkoeln.de.

** Der Autor dankt den Teilnehmern der GIRA-Jahrestagung am 22. und 23. Oktober 2015 und den anonymen Gutachtern für wertvolle Hinweise und Anregungen.

Artikel eingegangen: 29.2.2016

revidierte Fassung akzeptiert nach doppelt-blindem Begutachtungsverfahren: 13.6.2016. 
we developed a new method for measuring the intensity of conflict during collective bargaining which not only considers material measures but also verbal and formal measures like strike threats, termination of negotiations or arbitrations. These measures are ranked in an escalation pyramid. In a subsequent step, we derived two measures of escalation: As a static measure, the maximum escalation level informs about the maximum level, to which a single conflict escalates. As a dynamic measure, the intensity of conflict summarizes all conflict actions. An evaluation of 144 wage disputes in 13 industries shows significant differences in both measures between industries. Furthermore, we identified four patterns of conflict resolution. They indicate that collective bargaining in Germany can still be described by "partnership in conflict" (Konfliktpartnerschaft). In this regard, we can distinguish between a verbal-formal pattern of conflict resolution and a material pattern.

Key words: collective bargaining, intensity of conflict, industrial action, social partnership (JEL: J51, J52, J53)

\section{Problemstellung}

Die Stabilität der Arbeitsbeziehungen stellt einen wichtigen Standortvorteil dar. Streiks verursachen nicht nur gesamtwirtschaftliche Wohlfahrtsverluste. Ein hohes Maß an sozialem Frieden schafft einer vernetzten Volkswirtschaft stabile Rahmenbedingungen. Waren die Arbeitsbeziehungen in Deutschland im 19. Jahrhundert noch durch einen „Klassenkampf" geprägt, wandelte sich das Verhältnis zwischen den Tarifparteien im Laufe des 20. Jahrhunderts. Mit der Anerkennung der Gewerkschaften durch die Arbeitgeber im StinnesLegien-Abkommen vom November 1918 entstand die Grundlage für die Entwicklung einer Tarifpartnerschaft (Lesch, 2016). Walther Müller-Jentsch (1999; 2013) prägte dafür den Begriff „Konfliktpartnerschaft“. Mit der Kombination der gegensätzlichen Begriffspaare „Konflikt“ und „Partnerschaft“ will er ausdrücken, dass die Akteure der industriellen Beziehungen unterschiedliche Interessen vertreten, aber eine Schnittmenge gleicher Ziele haben. Diese Schnittmenge ist es, die die Akteure dazu veranlasst, miteinander zu kooperieren - mal konfliktreicher, mal konfliktärmer. Streeck (2016) hat den Begriff „Konfliktpartnerschaft“ nun für das 21. Jahrhundert in Frage gestellt und von einer „konfliktfrei gehaltenen Partnerschaft“" in der deutschen Exportindustrie auf der einen Seite und einem „Konflikt ohne Partnerschaft" in vielen Dienstleistungssektoren auf der anderen Seite gesprochen.

Dieses Ringen um Begrifflichkeit zeigt die Notwendigkeit auf, die Tarifpartnerschaft und den Lohnfindungsprozess einer tieferen empirischen Analyse zu unterziehen. Konkret stellt sich die Frage, wie sich das „Klima“ in den Tarifverhandlungen messen, beschreiben und gegebenenfalls systematisieren lässt. Ein Ansatz könnte darin bestehen, Experten zu befragen und Fallstudien durchzuführen. Ein alternativer, hier vorgezogener Ansatz versucht, Tarifpartnerschaft anhand objektiver Kriterien zu analysieren. In der Literatur wird die Qualität der Arbeitsbeziehungen anhand verschiedener Streikindikatoren gemessen (Gärnter 1989; Aligisakis 1997; Bordogna \& Cella 2002; Lesch 2002; Vandaele 2011; Carley 2013). ${ }^{1}$ Das gebräuchlichste Messkonzept ist die Anzahl der in einer Volkswirtschaft durch Streiks und Aussperrungen verlorenen Arbeitstage (Arbeitskampfvolumen).

1 Zur kritischen Diskussion der Streiktätigkeit als „Militanzindikator“ einer Gewerkschaft vgl. den Überblick bei Schnabel (1989, $63 \mathrm{ff}$.) 
Weitere Indikatoren sind die Streikhäufigkeit, der Streikumfang und die Streikdauer. Die Streikhäufigkeit informiert darüber, wie viele Konflikte in einer Periode aufgetreten sind, der Streikumfang gibt an, wie viele Beschäftigte sich im Durchschnitt an einem Ausstand beteiligten und die Streikdauer misst, wie lange ein Einzelner im Durchschnitt die Arbeit aus Protest niederlegte. Das Ergebnis von Arbeitskampfvergleichen auf Basis dieser Indikatoren lautet: Wo viele Ausfalltage anfallen und oft gestreikt wird, sind die Arbeitsbeziehungen konfliktreich, wo weniger Ausfalltage anfallen und eher selten gestreikt wird, herrscht sozialer Friede.

Die Analyse von Arbeitskampfindikatoren erlaubt demnach, zwischen konfliktreichen und konfliktarmen Arbeitsbeziehungen zu unterscheiden. Dies ist aber zu wenig differenziert, um Begriffe wie „Konfliktpartnerschaft“ oder „Konflikt ohne Partnerschaft“ auszuleuchten. Dazu ist eine detailliertere Betrachtung aller in Tarifverhandlungen genutzten Eskalationsformen notwendig. Sie ermöglicht es, unterschiedliche Konfliktlösungskulturen zu identifizieren und dadurch einen wichtigen Beitrag zur begrifflichen Klärung der Typen von Tarifpartnerschaft zu leisten.

Im Folgenden wird dazu ein neuer methodischer Ansatz mit empirischen Befunden vorgestellt. Kapitel 2 stellt nach einigen Bemerkungen über die Berücksichtigung der „Konflikttheorie“ in der ökonomischen Analyse eine Eskalationspyramide vor, die eine Hierarchie zwischen den bei Tarifverhandlungen üblichen Eskalationsformen herstellt. Darauf aufbauend werden zwei neue Indikatoren zur Messung des Konfliktpotenzials (der Konflikteskalation) beschrieben: die maximale Eskalationsstufe und die Konfliktintensität. Kapitel 3 stellt einige nach Branchen differenzierte empirische Befunde zu diesen beiden Indikatoren dar, arbeitet exemplarisch den Informationsgewinn gegenüber dem herkömmlichen Indikator Arbeitskampfvolumen heraus und identifiziert unterschiedliche Konfliktlösungskulturen. In Kapitel 4 werden die Empirie kurz mit der neuen Kontroverse zur „Konfliktpartnerschaft" (Streeck, 2016) in Verbindung gebracht, einige kritische methodische Bemerkungen im Rahmen der GIRA-Jahrestagung 2015 aufgegriffen und Anstöße zu einer Weiterentwicklung gegeben.

\section{Eskalationspyramide, maximale Eskalationsstufe und Konfliktintensität}

\subsection{Allgemeine Überlegungen}

Die sozialwissenschaftliche Konfliktforschung besteht aus einer Vielzahl unterschiedlicher Konflikttheorien, die sich in ihrem Selbstverständnis in normative, deskriptive oder analytische Konflikttheorien unterschieden lassen (Bonacker, 2008, S. 25). Dabei versuchen normative Ansätze, Konfliktregelungsinstrumente zu finden. Deskriptive Ansätze beschreiben Konflikte und analytische Ansätze erklären sie. Darüber hinaus gibt es eine Unterscheidung konflikttheoretischer Felder (Bonacker, 2008, S. 18). Es gibt Konflikttheorien im Kontext der Theorien internationaler Beziehungen, im Kontext von Gesellschaftstheorien und im Kontext akteurstheoretischer Ansätze. Akteurstheoretische Ansätze stellen die Frage, warum Akteure konflikthaft handeln (Bonacker, 2008). Die Rational-Choice-Theorie geht hierbei von der „Vorstellung zielorientierten Handelns“ (Kunz, 2008, S. 461) aus und folgt 
dabei dem Erklärungsbegriff der analytischen Wissenschaftstheorie. Dabei hat sich durchgesetzt, Konflikte auf der Basis individueller Handlungen spieltheoretisch zu modellieren (Kunz, 2008, S. 463).

Rationales Handeln und Entscheiden ist auch ein Kernelement in ökonomischen Ansätzen zur Erklärung von Tarifkonflikten. Diese Grundannahmen akteurstheoretischen Verhaltens stellen daher eine Brücke zwischen der sozialwissenschaftlichen und der wirtschaftswissenschaftlichen Debatte dar. Dabei gibt es auch in der Wirtschaftswissenschaft verschiedene Ansätze, die analysieren, warum Tarifverhandlungen nicht immer konfliktfrei ablaufen (Ross \& Hartmann 1960; Hicks 1963; Ashenfelter \& Johnson 1969; Külp 1969; Zerche 1970; Zerche 1979; Siebert \& Addison 1981; Schnabel 1989; Lesch 2013b). Neben den Theorien der Kollektivverhandlungen („Collective Bargaining“ Theorien) ist vor allem die Spieltheorie zu nennen. In spieltheoretischen Ansätzen wird eine Parallele zwischen Tarifverhandlungen und einem „Game of Chicken“ gezogen (Posner 2007, S. 346). Beide Tarifparteien stellen Maximalforderungen auf. Sofern keine Seite nachgibt, kommt es zum Arbeitskampf. Da dieser beiden Seiten schadet, haben beide einen Anreiz, die Eskalation zu vermeiden und eine Verhandlungslösung vorzuziehen. In der langen Frist wirken jedoch weitere Anreizmechanismen. Sofern der Arbeitskampf hilft, die Glaubwürdigkeit der eigenen Verhandlungsposition zu stärken, kann er durchaus im Interesse einer Partei liegen. Die Arbeitgeber müssen die Gewerkschaften disziplinieren, indem sie glaubwürdig drohen, im Falle unerwartet steigender Lohnkosten die Beschäftigung zu drosseln, die Produktion zu verlagern oder gar ganz einzustellen. Die Gewerkschaften müssen ihre Streikbereitschaft und Streikfähigkeit hinreichend beweisen. Dazu kann eine Streikdrohung durchaus ausreichen. Ist eine solche aber unglaubwürdig, muss die Gewerkschaft ihre Glaubwürdigkeit durch einen Streik wiederherstellen. Nach Hicks (1963) muss dies sogar regelmäßig geschehen. Letztlich haben beide Seiten einen Anreiz, die kurzfristigen Folgen eines Arbeitskampfes hinzunehmen, um langfristig die eigene Verhandlungsposition zu verbessern. Posner (2007, S. 346) spricht von „Reputationsgewinnen“, die die Machtbalance zwischen Tarifparteien beeinflussen, und damit auch den Anteil, den sich beide an der Verteilungsmasse sichern können.

Die in Tarifverhandlungen üblichen Eskalationsstufen werden in den ökonomischen Verhandlungs- und Streiktheorien nur teilweise berücksichtigt und analysiert. So weist Keller (1975, S. 118) darauf hin, dass Theorien der Kollektivverhandlungen normalerweise die Frage in den Vordergrund stellen, wie es zu einer Einigung kommt. Ein Scheitern der Verhandlungen stellt hingegen einen Sonderfall dar, der in der Regel nicht weiter betrachtet werde. In spieltheoretischen Lösungen des Verhandlungsproblems wurde neben den Drohungen auch die Rolle von Schiedsrichterlösungen berücksichtigt (Zerche, 1970, S. 122 f.; Zerche 1979, S. 99 ff.). Allerdings steht auch dort im Vordergrund, dass der Unparteiische eine Einigung herbeiführt. Es geht weniger darum, wie er es tut und welche Anreizwirkungen auf die Tarifparteien bestehen. Vor diesem Hintergrund überrascht nicht, dass es bislang keine empirische Evidenz darüber gibt, welche Rolle die einzelnen Eskalationsstufen in den Tarifverhandlungen tatsächlich spielen.

\subsection{Konflikthandlungen}

Bei der nachstehend vorgestellten neuen Methode zur Messung der Konflikteskalation in Tarifauseinandersetzungen werden alle verhandlungsbegleitend ergriffenen verbal-formalen 
und materiellen Konflikthandlungen berücksichtigt. Materielle Konflikthandlungen, sind Warnstreiks, Streiks und Aussperrungen. Sie sind in dem Sinne materiell, als sie gezielt dazu benutzt werden, Produktionsausfälle zu verursachen. Verbale Konflikthandlungen sind Streik- oder Aussperrungsdrohungen, konkrete Streik- oder Aussperrungsankündigungen sowie die offizielle Erklärung einer Tarifpartei, dass die Tarifverhandlungen gescheitert sind. Formale Konflikthandlungen sind Verhandlungsabbrüche, Schlichtungen, juristische Auseinandersetzungen und Urabstimmungen. Zunächst werden die einzelnen Konflikthandlungen erläutert. Danach werden sie im Hinblick auf ihr Zuspitzungspotenzial in eine Reihenfolge gebracht. Dies geschieht mit Hilfe einer Eskalationspyramide (Lesch, 2013a).

Da die Tarifparteien in Tarifverhandlungen unterschiedliche Interessen vertreten, stellen sie in der Regel auch unterschiedliche Forderungen auf und bewerten einzelne Forderungen auch unterschiedlich. Um zu einem Kompromiss zu gelangen, sind Verhandlungen notwendig. In deren Rahmen können die beiden Tarifparteien ihre Positionen darlegen, begründen und für sie werben. Die Verhandlungen sind notwendig, um Informationsasymmetrien abzubauen. Beide Seiten können so ausloten, wo die Kompromisslinie des jeweiligen Verhandlungspartners liegt. Tarifverhandlungen sind demnach eskalationsfreie Maßnahmen zur Befriedung eines Tarifkonflikts.

Bei vollständiger Information müssten rational handelnde Tarifparteien die jeweilige Kompromisslinie des Verhandlungsgegners erkennen. Es stellt sich aber die bereits in Kapitel 2.1 kurz erörterte Frage, ob diese Kompromisslinie exogen oder endogen ist. Ist sie endogen, haben beide Seiten einen Anreiz, die Kompromisslinie des Gegners zu beeinflussen. Dieser Einfluss muss nicht unbedingt über materielle Konflikthandlungen ausgeübt werden. Denkbar sind auch verbale und formale Drohungen. ${ }^{2}$ Die Streikdrohung spielt schon in der Lohntheorie von Hicks (1963) eine wichtige Rolle. Die Arbeitgeber stehen vor jeder Verhandlungsrunde vor dem Problem, dass ein Eingehen auf die Gewerkschaftsforderungen die Arbeitskosten erhöhen, sie im Falle eines Ablehnens aber Gefahr laufen, bestreikt zu werden. Die Gewerkschaften müssen damit rechnen, dass sie im Fall eines frühzeitigen Kompromisses auf Lohnerhöhungen verzichten, die sie vielleicht bei weiteren Streikdrohungen hätten erzielen können. Lehnen sie hingegen Arbeitgeber-Offerten ab, müssen sie mit einem Abbruch der Verhandlungen rechnen und tatsächlich zum Streik aufrufen (Külp, 1969, S. 39 ff.; Zerche, 1970, S. 38 ff.). Letztlich bestimmen die erwarteten Streikkosten den Kompromiss. Je größer die erwarteten Streikkosten, desto eher lassen sich die Arbeitgeber auf höhere Löhne ein. Die Streikdrohung erhöht als verbale Vorstufe des Streiks das Risiko eines Arbeitskampfs und damit auch den Erwartungswert der Streikkosten.

Üblicherweise verabreden die Tarifparteien nach einer Tarifverhandlungsrunde entweder einen neuen Verhandlungstermin, oder sie signalisieren der Gegenseite, dass sie im Gespräch bleiben wollen. Wird eine Verhandlung aber im Dissens ohne Vereinbarung eines neuen Termins (oder ohne ein Signal, sich möglichst schnell wieder am Verhandlungstisch treffen zu wollen) vertagt, liegt ein Verhandlungsabbruch vor. Er stellt eine erste formale Stufe der Eskalation dar, ist aber noch kein offizielles Scheitern der Tarifverhandlungen.

2 So stellt Külp (1969, S. 8) fest: „Dass die Tarifparteien ihre Verhandlungen zu ihren Gunsten zu beeinflussen versuchen, indem sie mit Streik und Aussperrung drohen, bedarf wohl keiner besonderen Erwähnung.“ 
Grundsätzlich lässt der Abbruch die Möglichkeit zu, die Verhandlungen nach Sondierungen wieder aufzunehmen. ${ }^{3}$

Eine weitere verbale Eskalationsstufe liegt vor, wenn eine Gewerkschaft zum Streik aufruft oder ein Arbeitgeber aussperren will. Ein Streikaufruf ist möglich, sobald die tarifliche Friedenspflicht endet. ${ }^{4}$ Für die Aussperrung gelten restriktivere Regeln. Die Rechtsprechung lässt Aussperrungen nur in Reaktion auf einen Streik zu (Rieble, 2005; Berg, Platow, Schoof, \& Unterhinninghofen, 2010, S. 597). Im Falle eines Streikaufrufs informiert eine Gewerkschaft den Arbeitgeber und gegebenenfalls auch die Öffentlichkeit darüber, dass zu einem bestimmten Zeitpunkt Arbeitskampfmaßnahmen ergriffen werden. Dabei kann es sich entweder um zeitlich befristete Arbeitskampfhandlungen handeln (Warnstreiks) oder um einen unbefristeten Streik (Erzwingungsstreik). Der Streikaufruf ist zunächst lediglich eine Verschärfung oder Konkretisierung der Streikdrohung. Es muss in Reaktion auf einen solchen Aufruf nicht zwingend zu einem Streik kommen. Häufig besteht noch ein Zeitfenster, das sich noch nutzen lässt, einen Arbeitskampf durch eine Wiederaufnahme von Verhandlungen zu vermeiden.

Wird ein solches Zeitfenster nicht genutzt, kommt es in der Regel nicht direkt zu einem unbefristeten Streik, sondern erst einmal zu einem zeitlich befristeten Warnstreik. Der Warnstreik ist bereits eine materielle Konflikthandlung, die auch zu Produktionsunterbrechungen oder Ausfällen führt und damit einen ökonomischen Schaden verursacht. Aus Sicht der Gewerkschaften soll der Warnstreik eine weitere Eskalation in Form eines Scheiterns der Verhandlungen verhindern, die eine Urabstimmung und einen unbefristeten Streik nach sich ziehen kann (Lesch, 2013a, S. 67). Nach Däubler (2011, S. 366) stellt der Warnstreik ein „Frühwarnsystem“ dar. Dies beruhe zum einen auf der Demonstrationsfunktion, zum anderen auf einer Mobilisierungsfunktion. Die Demonstrationsfunktion bestehe darin, die Ernsthaftigkeit der Forderungen ebenso zu verdeutlichen wie die Konfliktfähigkeit. Die Mobilisierungsfunktion liege darin, dass die befristete Arbeitsniederlegung der Gewerkschaftsführung zeige, wie gut sie ihre Mitglieder für einen eventuell unbefristet geführten Arbeitskampf mobilisieren kann.

Eine weitere Verschärfung liegt vor, wenn eine Tarifpartei erklärt, die Tarifverhandlungen seien gescheitert. Mit dieser finalen verbalen Eskalationsform gibt die erklärende Partei zu verstehen, dass alle bilateralen Verhandlungsmöglichkeiten ergebnislos ausgeschöpft wurden. Das Scheitern zieht in der Regel ein bestimmtes formales Verfahren nach sich. Entweder bestehen Schlichtungsvereinbarungen, die nach dem Scheitern eine Schlichtung vorsehen, oder die Gewerkschaften leiten eine Urabstimmung ein. Ist weder eine Schlichtung noch eine Urabstimmung vorgesehen, kann eine Gewerkschaft (unter Beachtung der tariflichen Friedenspflicht) auch unmittelbar streiken (Däubler, 2011, S. 285).

Die unterschiedlichen Verfahren bedeuten, dass zwischen der Eskalationsform Scheitern und Schlichtung auf der einen Seite und der Eskalationsform Scheitern und Urabstim-

3 Sondierungen sind ein regelmäßig genutztes Instrument in Tarifverhandlungen. Da sie eine Art ,inoffizielle Verhandlung“ im kleinen Kreis darstellen, werden sie nicht als eigene Handlung berücksichtigt, sondern dort, wo es angebracht ist, unter Tarifverhandlung verortet.

4 Die Friedenspflicht endet, sobald ein Tarifvertrag außer Kraft tritt. Die Tarifparteien können die Friedenspflicht aber durch Schlichtungsabkommen oder durch eine vereinbarte Nachfrist verlängern (Berg et al., 2010, S. 574). In der Metall- und Elektro-Industrie endet die Friedenspflicht vier Wochen nach dem Ablauf des Tarifvertrags. 
mung oder Scheitern und Streik auf der anderen Seite zu unterscheiden ist. Die Schlichtung ist ein Verfahren zur Beilegung kollektiver Regelungsstreitigkeiten zumeist durch Intervention eines am Konflikt unbeteiligten Dritten (Keller, 1985, S. 119). Konfliktsoziologisch wird die Vereinbarung von Schlichtungsordnungen als wesentliches Element des „Machtausgleich[s] durch die Institutionalisierung von Konfliktzonen“" interpretiert (Keller, 1985, S. 120). Die faktisch vorhandenen industriellen Konflikte sollen durch die Festsetzung von langfristig geltenden Verfahrensregeln und durch die Einbeziehung unabhängiger Instanzen befriedet werden. Untersuchungen über die Wirkung der Schlichtung haben gezeigt, dass die Funktion des Schlichters vor allem darin besteht, die Qualität der vorhandenen Informationen so zu beeinflussen, dass er ihnen eine größere Sicherheit verleiht (Külp, Keller, Wiedemann, \& Wolters, 1972, 29). Allerdings kann eine Schlichtung auch als integraler Bestandteil der Verhandlungstaktik benutzt werden (Keller, 1985, S. 123). Dadurch können beabsichtigte Konzessionen aufgeschoben werden (Külp et al., 1972, S. 25; Keller, 1985, S. 126). In diesem Fall würde ein Konflikt verlängert - mit der Folge, dass auch einzelne Eskalationsformen wie Streikdrohungen häufiger vorkommen können.

Die Eskalationsform Scheitern und Urabstimmung (alternativ: Scheitern und Streik) kann - zeitlich betrachtet - entweder vor oder nach einer Schlichtung auftreten. Gilt eine Schlichtungsvereinbarung, darf die Gewerkschaft eine Urabstimmung oder den Streik erst einleiten, wenn die Schlichtung gescheitert ist. Im Falle einer Urabstimmung entscheiden die Mitglieder darüber, ob die tarifpolitischen Ziele mit Hilfe eines Streiks durchgesetzt werden sollen. Eine Urabstimmung stellt für die Gewerkschaft eine Streikhürde dar (Crampton, Gunderson, \& Tracy, 1999, S. 478). Dies liegt vor allem daran, dass Informationsasymmetrien abgebaut werden (Lesch, 2013b, S. 60). Für die Gewerkschaften ist zudem wichtig, dass die Urabstimmung den Arbeitskampf gegenüber der Öffentlichkeit legitimiert.

Eine weitere formale Konflikthandlung ist die juristische Auseinandersetzung. Sie tritt in verschiedenen Varianten und zu verschiedenen Zeitpunkten auf (Lesch, 2013a, S. 67). Häufig will eine Tarifpartei eine einstweilige Verfügung gegen den Gegner erwirken. Die Arbeitgeber wollen dadurch Streiks vermeiden, die Gewerkschaften wenden sich gegen Maßnahmen zum Streikbruch. Es gibt aber auch weitergehende juristische Auseinandersetzungen wie Schadensersatzklagen oder Klagen gegen den tariflichen Status. Bei Spartengewerkschaften stemmten sich beispielsweise die Arbeitgeber mit allen juristischen Mitteln dagegen, eine erstmals nach tarifpolitischer Eigenständigkeit strebende Gewerkschaft, die nur einzelne Berufe oder wenige Berufsgruppen vertritt, als tariffähige Organisation anzuerkennen und einer Aufnahme von Tarifverhandlungen zuzustimmen. Mit der juristischen Auseinandersetzung verlassen die Tarifparteien den bilateralen Konfliktraum. Wie bei der Schlichtung, wird eine dritte Instanz einbezogen. Während die Schlichtung auf eine Beilegung des Konflikts im Wege der Kompromissfindung setzt, zielt die juristische Auseinandersetzung auf eine Beschränkung der Handlungsmöglichkeiten ab, im Extremfall sogar auf die Vernichtung des Gegners. Letzteres wäre der Fall, wenn eine Gewerkschaft tatsächlich schadensersatzpflichtig wäre und dadurch insolvent würde oder einer Gewerkschaft die Tariffähigkeit abgesprochen würde.

Zum Arbeitskampf gehören Streik und Aussperrung. Der Streik ,ist die schärfste Waffe der Arbeitnehmer, ihre Ziele durchzusetzen“ (Zerche, 1979, S. 33). Es handelt sich um die gemeinsame, planmäßige Arbeitsniederlegung von Arbeitnehmern zur Erreichung eines 
bestimmten Kampfziels. Die Aussperrung war früher das schärfste Kampfmittel der Arbeitgeber, ist in Deutschland aber unüblich geworden. Bei diesem Instrument verweigern die Arbeitgeber den Arbeitnehmern den Zugang zum Arbeitsplatz ohne Fortzahlung des Arbeitslohns. Beide Eskalationsformen sind - ebenso wie der Warnstreik - materielle Konflikthandlungen, weil sie darauf abzielen, dem Gegner einen finanziellen Schaden zuzufügen, um ihn auf diese Weise zur Konzession zu zwingen.

\subsection{Eskalationspyramide}

Die verschiedenen Konflikthandlungen lassen sich mit Hilfe einer Eskalationspyramide so anordnen, dass sie die Zuspitzung von Tarifkonflikten abbilden (Lesch, 2013a). Dies ist in Abbildung 1 dargestellt. Die Positionierung einer Konflikthandlung innerhalb der Eskalationspyramide hängt nicht unbedingt mit ihrer zeitlichen Verortung zusammen. Zeitlich betrachtet beginnt ein Tarifkonflikt mit Forderungen und Gegenforderungen. Im Rahmen von Tarifverhandlungen werden Angebote unterbreitet und nach einem Kompromiss gesucht. Gelingt dies, ist der Konflikt beendet. Die Verhandlungen können von verbalen Konflikthandlungen wie Drohungen und Streikankündigungen oder von formalen Konflikthandlungen wie dem Verhandlungsabbruch begleitet sein. Wann die Konflikthandlungen genutzt werden, hängt - unter der Beachtung der Friedenspflicht - von der (autonomen) Entscheidung der Tarifparteien ab. Der Verhandlungsablauf ist hingegen determiniert, sobald eine Partei das Scheitern erklärt hat. In diesem Fall greifen entweder Schlichtungsvereinbarungen oder Satzungsbestimmungen. Die Schlichtung überführt den Konflikt wieder in das Verhandlungsstadium. Scheitert die Schlichtung oder ist eine solche nicht vereinbart, sehen viele Satzungen eine Urabstimmung vor. Sprechen sich die Gewerkschaftsmitglieder dabei für einen Streik aus, folgt der Arbeitskampf. Auch in diesem Fall müssen die Tarifparteien die Verhandlungen aber irgendwann wieder aufnehmen.

Das Festlegen von Eskalationsstufen setzt am zeitlichen Auftreten einer Konflikthandlung an, berücksichtigt aber auch deren Eskalationswirkung. Danach ist eine Streik- oder Aussperrungsdrohung ,eine erste und schwächste Stufe der Eskalation“ (Lesch, 2013a, S. 66). Anders als eine Streikankündigung beinhaltet die Streikdrohung noch keine Konkretisierung. Sie unterstreicht lediglich die Bereitschaft zum Streik, ohne die Verhandlungen zu unterbrechen. Die zweite Eskalationsstufe ist der Verhandlungsabbruch. Er stellt ebenfalls noch keine konkrete Streikgefahr dar, unterbricht aber den Verhandlungsprozess. Die Verhandlungen werden nicht mehr automatisch fortgesetzt, sondern erst nach einer „Abkühlungsphase“ mit entsprechenden Sondierungen. Die dritte Eskalationsstufe ist der Streikaufruf. Die Ankündigung eines Streiks ist nicht nur die Konkretisierung einer Streikdrohung. Sie verursacht in Branchen wie dem Verkehrssektor auch einen wirtschaftlichen Schaden.

Die vierte Eskalationsstufe ist der Warnstreik. Warnstreiks werden meist verhandlungsbegleitend eingesetzt, also bevor eine Verhandlung für gescheitert erklärt wurde. Es entsteht ein überschaubarer materieller Schaden, der als Warnsignal dient. Die Gewerkschaft nimmt dies bewusst in Kauf, um größere Schäden zu vermeiden, die durch unbefristete Streiks entstehen würden. Häufig reichen Warnstreiks als Druckmittel aus, um auf dem Verhandlungsweg einen Kompromiss zu erzielen. Ist dies nicht der Fall, eskaliert der Konflikt weiter, indem eine Seite das Scheitern der Verhandlungen erklärt. Zu diesem Zeitpunkt sind alle bilateralen Verhandlungsmöglichkeiten erschöpft. Zwei Eskalationsformen sind nun denkbar. 


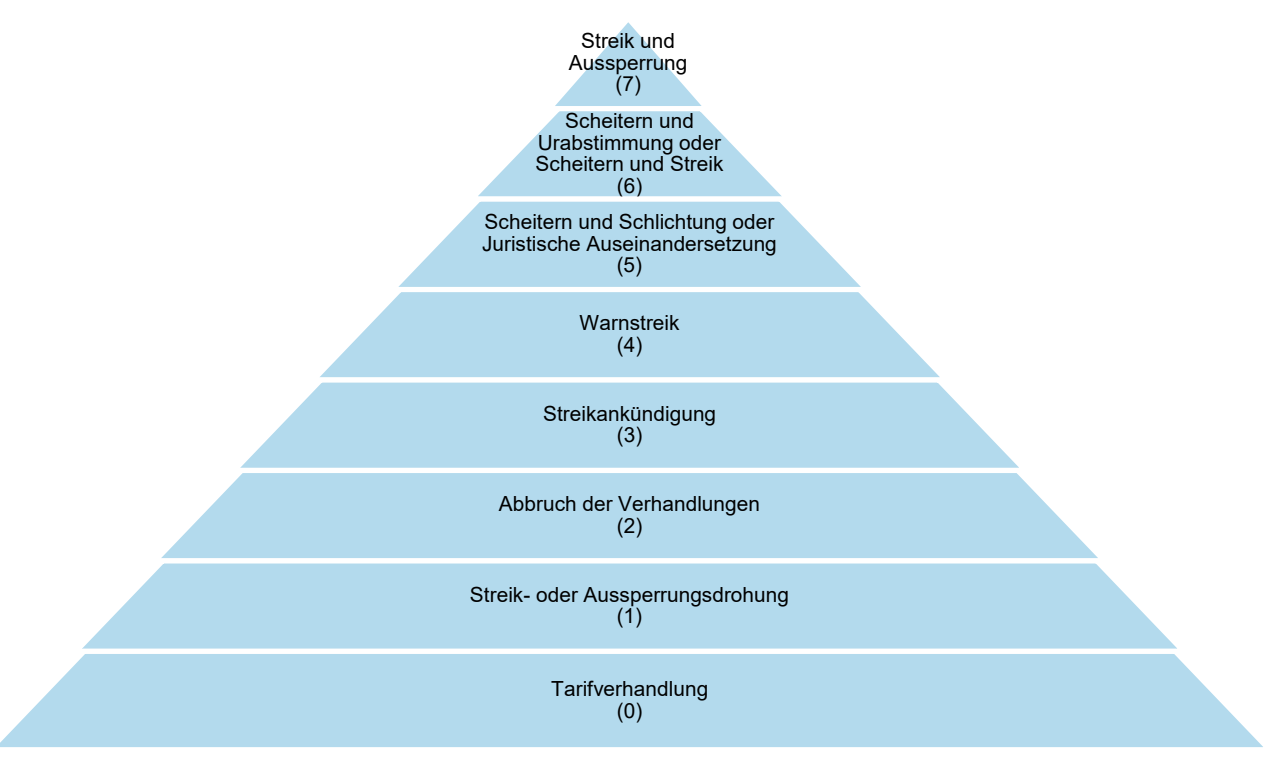

Abb. 1: Die Eskalationspyramide (Quelle: Lesch (2013a).

In der ersten Eskalationsform berufen die Tarifparteien einen neutralen Schlichter, dessen Aufgabe darin besteht, eine weitere Eskalation durch trilaterale Verhandlungen zu vermeiden. Eine erfolgreiche Schlichtung beendet den Konflikt, ohne dass ein materieller Schaden auftritt. Die Konflikthandlung Scheitern und Schlichtung wird als fünfte Eskalationsstufe (und damit eine Stufe höher als der Warnstreik) bewertet, obwohl sie eine neue Friedenspflicht begründet. Es darf während der Schlichtung weder zum Streik aufgerufen werden, noch ein Warnstreik organisiert werden. Es wäre daher denkbar, die Eskalationsform Scheitern und Schlichtung zwischen dem Verhandlungsabbruch und dem Streikaufruf zu verorten. Gegen diese Überlegung spricht, dass die Gewerkschaften den Warnstreik als Druckmittel nutzen, um sich bilateral mit den Arbeitgebern zu einigen. Er ist nicht darauf angelegt, den Konflikt trilateral im Wege der Schlichtung zu lösen. Verhandlungstaktisch ist die Schlichtung dem Warnstreik demnach nachgelagert. Im Ablaufschema der Tarifverhandlung ist die Schlichtungsphase ebenfalls einem relativ späten Stadium zuzuordnen (Keller, 1985, S. 119). In der Regel finden Warnstreiks statt, bevor das Scheitern der Verhandlungen erklärt wird und ein Schlichtungsmechanismus greift. Der Warnstreik will ein Scheitern ja gerade verhindern. Daher stellt das Scheitern gegenüber dem Warnstreik auch dann eine Eskalationsverschärfung dar, wenn es mit einer Schlichtung verbunden ist.

Ist keine Schlichtung vorgesehen, zieht das Scheitern eine unmittelbare oder eine mittelbare Ankündigung von Streiks nach sich. Dies stellt eine sechste Eskalationsstufe dar. Im unmittelbaren Fall verzichtet die Gewerkschaft auf eine Urabstimmung, im mittelbaren holt sie sich das Mitgliedervotum ein. Beide Fälle signalisieren, dass ein Arbeitskampf unvermeidbar ist. Streik und Aussperrung sind dann die siebte und letzte Eskalationsstufe. Beide Arbeitskampfformen gehen mit einem materiellen Schaden einher. 
Als Konflikthandlung wurden in Kapitel 2.2 auch juristische Auseinandersetzungen bewertet. Da diese zu verschiedenen Verhandlungszeitpunkten denkbar sind, hilft eine zeitliche Verortung bei der Einstufung in die Eskalationspyramide nicht weiter. Ein weiteres Problem ist ihre unterschiedliche Wirkung. Eine Schadensersatzklage zielt wie der Arbeitskampf auf eine materielle Wirkung, die sogar die Existenz des Gegners gefährden kann. ${ }^{5}$ Eine erfolgreiche einstweilige Verfügung gegen einen Streikaufruf will einen materiellen Schaden hingegen verhindern. Eines haben die verschiedenen juristischen Auseinandersetzungen jedoch gemeinsam (Lesch 2013a, S. 67): Indem eine Verhandlungsseite Rechtsmittel einlegt, signalisiert sie, dass sie zu einer bilateralen Verständigung in der Streitsache nicht bereit ist. Außerdem werden Rechtsmittel eingelegt, wenn eine Partei das Gefühl hat, dass sich die Gegenseite nicht an die Spielregeln der Verhandlungen hält. In allen Fällen besteht eine gewisse Parallele zur Schlichtung. Beide Verfahren ziehen in Form von Richtern oder Schlichtern neutrale Personen heran, die im trilateralen Kontext vermitteln. Durch das Einbeziehen der Arbeitsgerichtsbarkeit wird ein Konflikt analog zur Schlichtung durch Dritte befriedet. Dies legt es nahe, die Eskalationswirkung von juristischer Auseinandersetzung und Schlichtung einheitlich zu bewerten.

\subsection{Eskalationsmaße}

Die Eskalationspyramide bringt die verschiedenen Konflikthandlungen in eine bestimmte Hierarchie, stellt selbst aber noch kein Eskalationsmaß dar. Um die Konflikteskalation zu messen, bedarf es einer Methodik, die das Auftreten einzelner oder mehrerer Konflikthandlungen anhand der siebenstufigen Rangskala erfasst und bewertet. Lesch (2013a, S. 67 f.) schlägt hierzu zwei Bewertungsverfahren vor, ein statisches und ein dynamisches.

Das statische Verfahren beschränkt sich darauf, für jeden Tarifkonflikt zu prüfen, bis zu welcher Stufe der Konflikt eskaliert. Diese maximale Eskalationsstufe berücksichtigt weder die Länge einer Auseinandersetzung, noch ob sich der Konflikt mehrfach neu entzündet und sich Eskalationsstufen dadurch wiederholen. Eskaliert ein Tarifkonflikt beispielsweise mehrfach bis zum Warnstreik, hat dies keinen Einfluss auf die maximale Eskalationsstufe. Die statische Messung bietet den Vorteil, dass sich bestimmte Konfliktmuster leichter abbilden lassen (Lesch, 2013a, S. 72 f.). In einigen Branchen sind über den Zeitverlauf hinweg recht stabile maximale Eskalationsstufen beobachtbar, aus denen sich spezifische Konfliktlösungskulturen ableiten lassen (vgl. Kapitel 3.3).

Das dynamische Verfahren berücksichtigt den zeitlichen Verlauf. Kommt es wiederholt zu einer Eskalation, wird dies bei der Messung berücksichtigt. Dadurch wird die tatsächliche Konfliktintensität gemessen. Um das Problem der Skalierung zu minimieren, bietet sich ein summarisches Bewertungsverfahren an. Hierbei werden die Punkte, die den verschiedenen Konflikthandlungen im Rahmen der Eskalationspyramide zugeordnet werden, addiert. Allerdings wird jede Eskalationsstufe zwischen zwei aufeinanderfolgenden Tarifverhandlungen nur einmal gezählt. Eine solche Bereinigung ist sinnvoll, um die Vergleichbarkeit

5 Beispielhaft sei auf die wiederholten Schadensersatzklagen von Fluggesellschaften und Flughafenbetreibern gegen die Gewerkschaft der Flugsicherung verwiesen. Hätten die Kläger Recht bekommen, hätte die Berufsgruppengewerkschaft Konkurs anmelden müssen. 
von Tarifverhandlungen mit unterschiedlichen Geltungsbereichen und verschiedenen Streikformen zu gewährleisten. ${ }^{6}$

Wie unterschiedlich die beiden Bewertungsverfahren ausfallen können, zeigt das folgende Beispiel. Dazu werden zwei Konflikte mit jeweils vier Verhandlungsrunden betrachtet (siehe Übersicht 1). In Konflikt 1 kommt es nach der dritten Verhandlungsrunde zu einer Streikdrohung, einer Streikankündigung und einem Warnstreik. In der vierten Runde einigen sich die Parteien. In Konflikt 2 wird bereits nach der ersten Verhandlung eine Drohung ausgesprochen. Nach der zweiten Verhandlung folgen eine zweite Drohung, ein Aufruf und ein Warnstreik. Dasselbe Eskalationsschema wiederholt sich auch nach der dritten Verhandlung, allerdings wird die Streikdrohung zweimal ausgesprochen. Die zweite dieser Drohungen wird als wiederholte Drohung nicht als Konflikthandlung gewertet. In der vierten Verhandlung kommt es dann zu einer Einigung. In beiden Konflikten ist die Stufe 4, der Warnstreik, die maximale Eskalationsstufe. Bei der dynamischen Messung ergeben sich hingegen deutliche Unterschiede. In Konflikt 1 summieren sich die einzelnen Konflikthandlungen auf 8 Punkte $(1+3+4)$, in Konflikt 2 auf 17 Punkte $(1+1+3+4+1+3+4)$. Die Konfliktintensität ist bei Konflikt 2 demnach mehr als doppelt so hoch wie bei Konflikt 1. Die maximale Eskalationsstufe bildet das tatsächliche Konfliktgeschehen daher nur begrenzt ab.

Übersicht 1: Beispiele zur Messung von maximaler Eskalationsstufe und Konfliktintensität

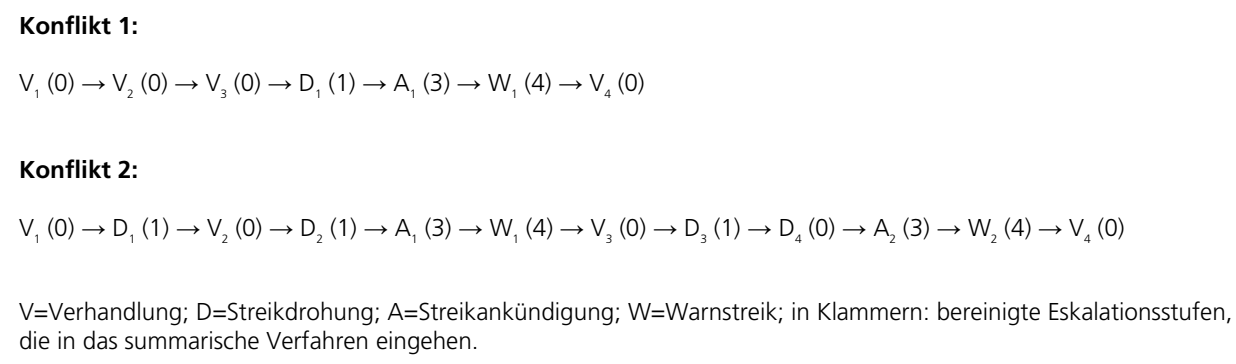

6 Neben unternehmensbezogenen Verhandlungen gibt es auch Branchenverhandlungen, die regional oder branchenweit geführt werden. Bei regionalen Verhandlungen können sich die einzelnen Eskalationsstufen allein dadurch wiederholen, dass sie verschiedene Tarifgebiete durchlaufen. Solche Wiederholungen scheiden bei bundesweiten Verhandlungen ebenso aus wie bei Verhandlungen für einzelne Unternehmen (Lesch 2013a, S. 68). Hinzu kommt, dass sich die Streikformen von Branche zu Branche unterscheiden. In einigen Dienstleistungssektoren wird ein unbefristeter Streik nach einer Urabstimmung eher kleinteilig geführt. Es kommt zu wiederholten, aber kürzeren Ausständen. Dazwischen gibt es auch Streikpausen. Wird in einem Tarifgebiet einer Industriebranche gestreikt, dauern die Aktionen in der Regel so lange an, bis die Parteien an den Verhandlungstisch zurückkehren. 


\section{Empirische Ergebnisse}

\subsection{Daten}

Grundlage der folgenden empirischen Auswertungen sind 144 Tarifkonflikte aus 13 Branchen seit dem Jahr 2000. Übersicht 2 fasst zusammen, welche Branchen ab welchem Zeitraum berücksichtigt wurden, wie die Tarifverträge strukturiert sind, welche Gewerkschaften und Arbeitgeberverbände miteinander verhandeln und wie viele Konflikte jeweils ausgewertet wurden. Die berücksichtigten Konflikte sind jeweils so präzise dokumentiert, dass der Konfliktbeginn (Datum der Tarifforderung oder Auslaufen eines Tarifvertrags), das Konfliktende (Tarifabschluss oder positive Urabstimmung nach einem Arbeitskampf) sowie die gewählten Konflikthandlungen bekannt sind. Die Dokumentation beginnt in einigen Branchen ab dem Jahr 2000, in anderen aber erst später. Grund ist die Fusion von fünf Einzelgewerkschaften zur Vereinten Dienstleistungsgewerkschaft ver.di 2001. In den betroffenen Branchen wurde jeweils mit der ersten ver.di-Tarifverhandlung begonnen.

Die Struktur der Tarifverträge unterscheidet sich zwischen den untersuchten Branchen. In den berücksichtigten Industriebranchen gilt ebenso wie im Bauhauptgewerbe, im privaten Bankgewerbe und im Einzelhandel ein Flächentarifvertrag. In Großunternehmen wie der Deutschen Telekom und der Deutschen Post gibt es Firmentarifverträge. Flächenund Firmentarifverträge beziehen sich auf alle Berufsgruppen. Daneben gibt es aber auch Unternehmen und Branchen mit Tarifverträgen für einzelne oder mehrere Berufsgruppen. Das gilt für die Deutsche Lufthansa, den Schienenverkehr, den Krankenhaussektor und den Öffentlichen Dienst. Im Schienenverkehr gibt es Firmentarifverträge, die von der EVG ausgehandelt werden und die für alle Berufsgruppen gelten. Daneben gibt es aber auch Tarifverträge, die nur für Lokführer oder das Zugpersonal gelten. Diese werden von der GDL abgeschlossen, die als Spartengewerkschaft nur bestimmte Berufsgruppen vertritt. Im Öffentlichen Dienst bestehen zwei Flächentarifverträge für die Länder und für die Tarifgemeinschaft des Bundes und der Kommunen. Daneben werden seit 2009 auch Spartentarifverträge (Entgeltrahmen-Tarifverträge) für die Berufsgruppen im Sozial- und Erziehungsdienst geschlossen. Dabei kam es für diese Berufsgruppen sowohl 2009 als auch 2015 zu zusätzlichen Lohnsteigerungen. 
Übersicht 2: $\quad$ Ausgewertete Tarifkonflikte

\begin{tabular}{|c|c|c|c|c|}
\hline Branche & $\begin{array}{l}\text { Struktur der } \\
\text { Tarifverträge }\end{array}$ & Gewerkschaften & Arbeitgeber & $\begin{array}{l}\text { Anzahl } \\
\text { Konflikte }\end{array}$ \\
\hline $\begin{array}{l}\text { Bauhauptgewerbe } \\
\text { (ab 2000) }\end{array}$ & Flächentarifvertrag & $\begin{array}{l}\text { IG Bauen-Agrar- } \\
\text { Umwelt }\end{array}$ & $\begin{array}{l}\text { Hauptverband der } \\
\text { Deutschen Bauindust- } \\
\text { rie; Zentralverband } \\
\text { Deutsches Baugewerbe } \\
\end{array}$ & 10 \\
\hline $\begin{array}{l}\text { Chemische Industrie } \\
\text { (ab 2000) }\end{array}$ & Flächentarifvertrag & $\begin{array}{l}\text { IG Bergbau, } \\
\text { Chemie, Energie } \\
\end{array}$ & \begin{tabular}{|l|} 
Bundesarbeitgeber- \\
verband Chemie
\end{tabular} & 12 \\
\hline $\begin{array}{l}\text { Druckindustrie } \\
(\mathrm{ab} 2002)\end{array}$ & Flächentarifvertrag & ver.di & $\begin{array}{l}\text { Bundesverband Druck } \\
\text { und Medien }\end{array}$ & 7 \\
\hline $\begin{array}{l}\text { Einzelhandel } \\
\text { (ab 2001) }\end{array}$ & Flächentarifvertrag & ver.di & \begin{tabular}{|l|} 
Handelsverband \\
Deutschland
\end{tabular} & 9 \\
\hline $\begin{array}{l}\text { Flugsicherung } \\
\text { (ab 2002) }\end{array}$ & Firmentarifvertrag & ver.di; GdF & $\begin{array}{l}\text { Deutsche Flugsiche- } \\
\text { rung (DFS) }\end{array}$ & $\begin{array}{c}14 \\
\text { (1 ver.di, } \\
13 \mathrm{GdF})\end{array}$ \\
\hline $\begin{array}{l}\text { Krankenhäuser } \\
\text { (ab 2002) }\end{array}$ & $\begin{array}{l}\text { Flächentarifvertrag; ab } \\
\text { 2005: Tarifverträge für be- } \\
\text { stimmte Berufe oder Be- } \\
\text { rufsgruppen }\end{array}$ & ver.di; MB & $\begin{array}{l}\text { Tarifgemeinschaft } \\
\text { deutscher Länder; Ver- } \\
\text { einigung der kommu- } \\
\text { nalen Arbeitgeberver- } \\
\text { bände } \\
\end{array}$ & $\begin{array}{c}22 \\
(11 \text { ver.di, } \\
11 \mathrm{MB})\end{array}$ \\
\hline $\begin{array}{l}\text { Luftfahrt } \\
\text { (ab 2001) }\end{array}$ & $\begin{array}{l}\text { Tarifverträge für bestimm- } \\
\text { te Berufe oder Berufs- } \\
\text { gruppen }\end{array}$ & ver.di; VC; UFO & Deutsche Lufthansa & $\begin{array}{c}23 \\
\text { (9 Ver.di, } \\
8 \text { VC, } \\
6 \text { UFO) }\end{array}$ \\
\hline $\begin{array}{l}\text { Metall- und } \\
\text { Elektro-Industrie } \\
\text { (ab 2000) }\end{array}$ & Flächentarifvertrag & IG Metall & $\begin{array}{l}\text { Südwestmetall; } \\
\text { Metall-NRW; Verband } \\
\text { der bayerischen M+E- } \\
\text { Industrie }\end{array}$ & 12 \\
\hline $\begin{array}{l}\text { Öffentlicher Dienst } \\
\text { (ab 2002) }\end{array}$ & $\begin{array}{l}\text { Flächentarifvertrag; ab } \\
2009 \text { Tarifverträge für be- } \\
\text { stimmte Berufe oder Be- } \\
\text { rufsgruppen }\end{array}$ & ver.di; GEW; dbb & $\begin{array}{l}\text { Tarifgemeinschaft } \\
\text { deutscher Länder; Ver- } \\
\text { einigung der kommu- } \\
\text { nalen Arbeitgeberver- } \\
\text { bände; Bundesinnen- } \\
\text { ministerium }\end{array}$ & 13 \\
\hline $\begin{array}{l}\text { Postdienste } \\
\text { (ab 2002) }\end{array}$ & Firmentarifvertrag & ver.di & Deutsche Post & 8 \\
\hline $\begin{array}{l}\text { Privates } \\
\text { Bankgewerbe } \\
\text { (ab 2001) }\end{array}$ & Flächentarifvertrag & ver.di & $\begin{array}{l}\text { Arbeitgeberverband } \\
\text { des Deutschen Bank- } \\
\text { gewerbes }\end{array}$ & 8 \\
\hline $\begin{array}{l}\text { Schienenverkehr } \\
\text { (ab 2000) }\end{array}$ & $\begin{array}{l}\text { Firmentarifvertrag; ab } \\
\text { 2003: Tarifverträge für be- } \\
\text { stimmte Berufe oder Be- } \\
\text { rufsgruppen }\end{array}$ & $\begin{array}{l}\text { EVG, GDL; bis No- } \\
\text { vember 2010: } \\
\text { Transnet/GDBA (Ta- } \\
\text { rifgemeinschaft), } \\
\text { GDL } \\
\end{array}$ & Deutsche Bahn & $\begin{array}{c}19 \\
\text { (2 Transnet/ } \\
\text { GDBA/GDL; } 6 \\
\text { Transnet/GDBA, } 4 \\
\text { EVG, 7 GDL) }\end{array}$ \\
\hline $\begin{array}{l}\text { Telekommunikation } \\
\text { (ab 2002) }\end{array}$ & Firmentarifvertrag & ver.di & Deutsche Telekom & 8 \\
\hline
\end{tabular}

Zahl der berücksichtigten Tarifkonflikte: 144; Stand: 15.02.2016; Einzelhandel: NRW; Krankenhäuser: ver.diTarifverträge des Öffentlichen Dienstes; dbb: Deutscher Beamtenbund und Tarifunion; EVG: Eisenbahn- und Verkehrsgewerkschaft; GDBA: Verkehrsgewerkschaft Gewerkschaft Deutscher Reichsbahnbeamten und Anwärter; GdF: Gewerkschaft der Flugsicherung; GDL: Gewerkschaft Deutscher Lokomotivführer; GEW: Gewerkschaft Erziehung und Wissenschaft; MB: Marburger Bund; Transnet: Gewerkschaft der Eisenbahner Deutschlands; UFO: Unabhängige Flugbegleiter-Organisation; VC: Vereinigung Cockpit; ver.di: Vereinte Dienstleistungsgewerkschaft. 


\subsection{Eskalationsmaße}

Übersicht 3 fasst die empirischen Befunde zu den beiden Eskalationsmaßen maximale Eskalationsstufe und Konfliktintensität sowie zur Verhandlungsdauer für die 13 untersuchten Branchen zusammen. ${ }^{7}$ Wo mehrere Gewerkschaften verhandelt haben, wurde jeweils der gewichtete Durchschnitt berechnet. Die Gewichte ergeben sich jeweils aus dem Anteil der geschlossenen Tarifverträge. Im Luftverkehr hat die Vereinigung Cockpit (VC) beispielsweise acht der insgesamt 23 Tarifverträge geschlossen, sodass die VC auf einen Anteil von knapp 35 Prozent kommt. Die maximale Eskalationsstufe liegt im Durchschnitt aller ausgewerteten Tarifkonflikte einer Branche zwischen 0,3 Punkten in der Chemischen Industrie und 5,0 Punkten im Einzelhandel. Vergleichsweise hohe maximale Eskalationsstufen weisen der Öffentliche Dienst (4,6 Punkte), die Telekommunikation (jeweils 4,4 Punkte), die Metall- und Elektro-Industrie (4,2 Punkte), die Krankenhäuser und die Druckindustrie (jeweils 4,0 Punkte) auf. Im privaten Bankgewerbe (2,4 Punkte), in der Flugsicherung (2,8 Punkte) und im Postdienst (3,1 Punkte) fallen die maximalen Eskalationsstufen erkennbar geringer aus. Sie liegen aber noch deutlich über dem Wert in der Chemischen Industrie.

Übersicht 3: Eskalationsmaße und Verhandlungslänge; Durchschnittswerte

\begin{tabular}{lccc}
\hline Branche & $\begin{array}{c}\text { Maximale Eskalationsstufe } \\
\text { (in Stufen) }\end{array}$ & $\begin{array}{c}\text { Konfliktintensität } \\
\text { (in Punkten) }\end{array}$ & $\begin{array}{c}\text { Verhandlungslänge } \\
\text { (in Monaten) }\end{array}$ \\
\hline Bauhauptgewerbe & 3,6 & 7,7 & 5,4 \\
Chemische Industrie & 0,3 & 0,3 & 2,1 \\
Druckindustrie & 4,0 & 16,1 & 6,6 \\
Einzelhandel & 5,0 & 18,4 & 7,0 \\
Flugsicherung & 2,8 & 7,2 & 6,3 \\
Krankenhäuser & 4,0 & 17,6 & 5,0 \\
Luftfahrt & 3,3 & 15,5 & 9,3 \\
Metall- und Elektro-Industrie & 4,2 & 19,9 & 3,5 \\
Öffentlicher Dienst & 4,6 & 21,3 & 5,3 \\
Postdienste & 3,1 & 15,1 & 2,2 \\
Privates Bankgewerbe & 2,4 & 8,0 & 5,5 \\
Schienenverkehr & 3,4 & 15,2 & 7,6 \\
Telekommunikation & 4,4 & 17,3 & 3,6 \\
\hline
\end{tabular}

Die Konfliktintensität reicht von 0,3 Punkten in der Chemischen Industrie bis zu 21,3 Punkten im Öffentlichen Dienst. Allerdings liegen neun der 13 Branchen in einer deutlich kleineren Spanne von 15,1 und 21,3 Punkten. Am oberen Ende liegen neben dem Öffentlichen Dienst auch die Metall- und Elektro-Industrie mit 19,9 Punkten und der Einzelhandel mit 18,4 Punkten. Eher konfliktarm ging es mit 7,2 über 7,7 bis 8,0 Punkten auch in der Flugsicherung, im Bauhauptgewerbe sowie im Privaten Bankgewerbe zu. Vergleicht man die Konfliktintensität mit der Verhandlungslänge, zeigt sich kein enger Zusammenhang.

7 Die Berechnungen sind nicht direkt mit denen von Lesch (2013a) oder Lesch \& Hellmich (2014) vergleichbar. Zum einen werden hier gewichtete (anstelle ungewichteter) Durchschnitte berechnet, zum anderen gibt es Unterschiede bei der Konfliktauswahl. So werden im Schienenverkehr ausschließlich die Deutsche Bahn und in der Flugsicherung nur die Deutsche Flugsicherung betrachtet. Außerdem werden im Schienenverkehr und im Krankenhaussektor andere Zeiträume betrachtet. 
Bei den neun Branchen mit der höchsten Konfliktintensität streut die Verhandlungslänge beträchtlich. In der Druckindustrie und in der Telekommunikation kommen im Durchschnitt in 3,6 Monaten insgesamt 17,3 Konfliktpunkte zusammen, während sich die 15,5 Punkte in der Luftfahrt über 9,3 Monate ansammelten. Dieser Vergleich zeigt: Wird länger verhandelt, muss das nicht unbedingt mit einer höheren Konfliktintensität einhergehen. Es kommt auch vor, dass zwischen Eskalationsphasen längere „Abkühlungsphasen“ liegen.

\subsection{Konfliktintensität versus Streikausfalltage}

Um die Unterschiede zwischen den Eskalationsmaßen und den Arbeitskampfindikatoren zu zeigen, müssen die Eskalationsmaße kalenderjährlich berechnet werden. Dazu werden wie bei den Ausfalltagen auch - die Konfliktpunkte jener Tarifauseinandersetzungen, die sich über einen Jahreswechsel hinaus hinziehen, den entsprechenden Kalenderjahren zugeordnet. Abbildung 2 stellt die jährliche Konfliktintensität (in Punkten) der Anzahl der arbeitskampfbedingt ausgefallenen Arbeitstage seit dem Jahr 2000 gegenüber. Dabei fallen zwei Punkte auf. Erstens entwickeln sich die beiden Indikatoren erstaunlich parallel. Den Spitzen bei den Ausfalltagen stehen auch Spitzen bei der Konfliktintensität gegenüber (2002, 2006, 2015). Bei den Tiefpunkten sieht es ähnlich aus (2000, 2004, 2010). Zweitens entwickelt sich die Konfliktintensität in der Phase eines geringeren Arbeitskampfvolumens (2008 bis 2014) weniger gleichmäßig. Das deutet an, dass es in Jahren mit vergleichsweise wenigen Ausfalltagen in Tarifverhandlungen trotzdem konfliktreich zuging. Der Grund hierfür ist, dass die Zahl der Ausfalltage sehr stark durch Großkonflikte beeinflusst wird.

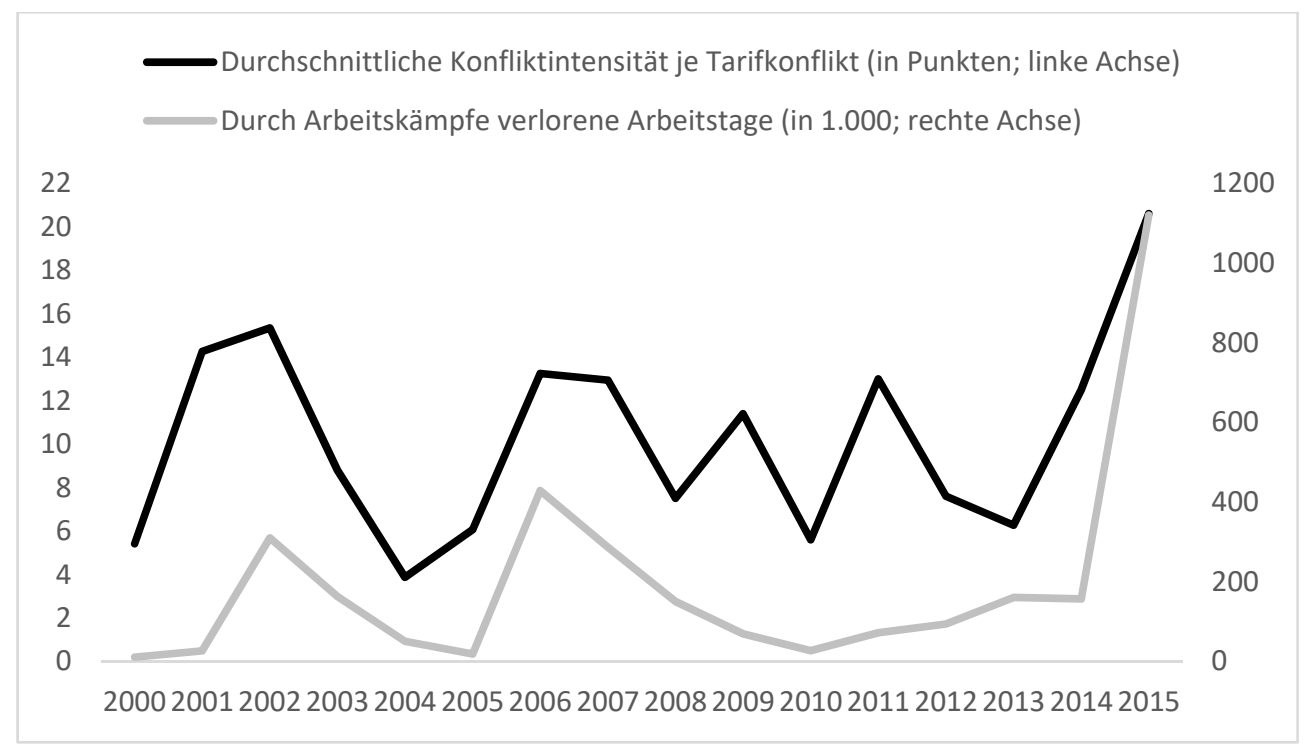

Abb. 2: Konfliktintensität und arbeitskampfbedingt verlorene Arbeitstage 2000 bis 2015

Dies wird durch einen exemplarischen Vergleich eines Spartentarifkonflikts mit einem Großtarifkonflikt deutlicher: dem Tarifkonflikt zwischen der Spartengewerkschaft GDL 
und der Deutschen Bahn 2014/15 und dem Konflikt zwischen der Multi-Branchengewerkschaft ver.di und der Deutschen Post 2015. Der Bahntarifkonflikt zog sich von Juni 2014 bis Anfang September 2015 über fast 15 Monate hin, der Posttarifkonflikt dauerte knapp 6 Monate (von Anfang 2015 bis Mitte 2015). Beide Konflikte eskalierten bis zum Arbeitskampf (Eskalationsstufe 7) und die Summe der einzelnen Konflikthandlungen betrug jeweils 73 Punkte (Übersicht 4). Beim Bahnkonflikt setzt sich diese Gesamtsumme aus acht Streikdrohungen (8 Punkte), sechs Verhandlungsabbrüchen (12 Punkte), vier Streikaufrufen (12 Punkte), einem Warnstreik (4 Punkte), einem Scheitern mit Schlichtung (5 Punkte), einer juristischen Auseinandersetzung (5 Punkte), einem Scheitern mit Urabstimmung (6 Punkte) und drei Streiks (21 Punkte) zusammen. Bei der Post gab es vier Streikdrohungen (4 Punkte), drei Streikaufrufe (9 Punkte), vier juristische Auseinandersetzungen (20 Punkte), ein Scheitern mit Urabstimmung (6 Punkte), fünf Warnstreiks (20 Punkte) und zwei Streiks (14 Punkte). ${ }^{8}$ Trotz unterschiedlicher Konflikthandlungen kommen beide Konflikte auf dieselbe Konfliktintensitätspunktzahl.

Übersicht 4: Konfliktintensität und Ausfalltage ausgewählter Tarifkonflikte

\begin{tabular}{lcccc}
\hline & $\begin{array}{c}\text { Konfliktintensität } \\
\text { (in Punkten) }\end{array}$ & $\begin{array}{c}\text { Maximale } \\
\text { Eskalationsstufe }\end{array}$ & $\begin{array}{c}\text { Verhandlungslänge } \\
\text { (in Monaten) }\end{array}$ & Ausfalltage \\
\hline Deutsche Bahn (GDL) & 73 & 7 & 14,5 & 64.000 \\
$\begin{array}{l}\text { Deutsche Post } \\
\text { (ver.di) }\end{array}$ & 73 & 7 & 5,5 & 550.000 \\
\hline
\end{tabular}

Ausfalltage: Schätzung auf Basis der amtlichen Streikstatistik und Streikteilnehmermeldungen der GDL.

Bei den Ausfalltagen bestehen allerdings große Unterschiede. Die amtliche Streikstatistik weist die beiden Unternehmen zwar nicht einzeln aus, sondern im Rahmen der Wirtschaftsgruppen „Landverkehr und Transport in Rohrfernleitungen“ sowie „Post-, Kurier- und Expressdienste“. Zusammen mit den von den Gewerkschaften veröffentlichten Streikteilnehmerzahlen lässt sich die Anzahl der Ausfalltage aber grob abschätzen. Bei der Deutschen Bahn waren es insgesamt etwa 64.000 Ausfalltage (2014: 40.000, 2015: 24.000), bei der Deutschen Post hingegen rund 550.000. Zwei Tarifauseinandersetzungen, die vergleichbar konfliktintensiv verliefen, führten demnach allein wegen ihrer Größe zu einer unterschiedlichen Anzahl an Ausfalltagen. Während bei der Post mehrfach bis zu 30.000 Arbeitnehmer im Ausstand waren, waren es bei der Bahn in der Regel nur 3.000. Aus diesem Beispiel folgt: Die Konfliktintensität kann auch dann hoch ausfallen, wenn es vergleichsweise wenige Ausfalltage gibt. Das ist vor allem in solchen Jahren der Fall, in denen mehrere Spartentarifkonflikte eskalieren, es aber keinen Großkonflikt gibt. Abbildung 2 zeigt: Das Jahr 2014 war im Hinblick auf die Ausfalltage durchaus unauffällig. Die Konfliktintensität lag aufgrund der mehrfach eskalierenden Spartentarifkonflikte bei Piloten und Lokführern aber recht hoch. Das bedeutet: Die Zahl der Ausfalltage wird viel stärker durch Großkonflikte dominiert als das alternative Eskalationsmaß Konfliktintensität. Umgekehrt wird die Konfliktintensität durch stark eskalierende Kleinkonflikte in die Höhe getrieben, während Spar- 
tentarifkonflikte in der Regel nur wenige Ausfalltage verursachen. Es ist deshalb sinnvoll, bei der Bewertung des Tarifklimas möglichst viele Indikatoren zu betrachten.

\subsection{Konfliktlösungskulturen}

Die in Kapitel 2.2 vorgestellten Konflikthandlungen wurden nicht nur in eine Eskalationshierarchie überführt. Sie wurden auch verbal-formalen und materiellen Eskalationsstufen zugeordnet. An diese Unterscheidung anknüpfend, ergeben sich drei unterschiedliche Ansätze oder Strategien, um Tarifkonflikte zu lösen:

- durch Verhandlung,

- durch verbal-formale Konflikthandlungen (Drohung, Verhandlungsabbruch, Streikaufruf, Schlichtung und Urabstimmung) und

- durch materielle Konflikthandlungen (Warnstreik, Streik und Aussperrung).

Jede dieser drei Strategien steht für eine bestimmte „Konfliktlösungskultur“. Um die jeweilige Bedeutung dieser drei Ansätze in den einzelnen Branchen zu bestimmen, werden ihre relativen Anteile anhand der maximalen Eskalationsstufe bestimmt. Der so bestimmte relative Anteil gibt an, wie wichtig die drei Ansätze zur Durchsetzung der tarifpolitischen Ziele in den einzelnen Branchen jeweils sind. Liegt der Anteil eines Ansatzes bei mindestens 50 Prozent und ist es zugleich der höchste Anteil, wird dieser als dominanter Verhandlungsansatz bezeichnet. ${ }^{9}$

Abbildung 3 stellt die Anteile der drei Ansätze für die 13 untersuchten Wirtschaftsbereiche dar. In Branchen oder Unternehmen, in denen mehrere Gewerkschaften verhandeln, wurden alle maximalen Eskalationsstufen berücksichtigt. Die Branchen sind in der Reihenfolge des Anteils der Verhandlungen als maximale Eskalationsstufe gereiht. Die Intention der Abbildung wird anhand eines Beispiels deutlicher. Im Bauhauptgewerbe wurden drei der insgesamt zehn Tarifkonflikte (30 Prozent) allein durch Verhandlungen gelöst, in fünf Fällen (50 Prozent) kam es (maximal) zu verbal-formalen Konflikthandlungen (einmal Verhandlungsabbruch und viermal Schlichtung) und in zwei Fällen (20 Prozent) zu materiellen Konflikthandlungen. Im Bauhauptgewerbe dominierten damit verbal-formale Konflikthandlungen. Das heißt: Jeder zweite Konflikt spitze sich bis zu verbal-formalen Konflikthandlungen zu.

9 Liegt beispielsweise der Anteil der Verhandlung bei 50 Prozent und liegen die Anteile der verbal-formalen und der materiellen Konflikthandlungen bei jeweils 25 Prozent, ist die Verhandlung die dominierende Konfliktlösungsstrategie. Liegt aber der Anteil der Verhandlung bei 50 Prozent und gleichzeitig auch der Anteil der materiellen Konflikthandlungen, ist die Verhandlung nicht die dominierende Strategie. 


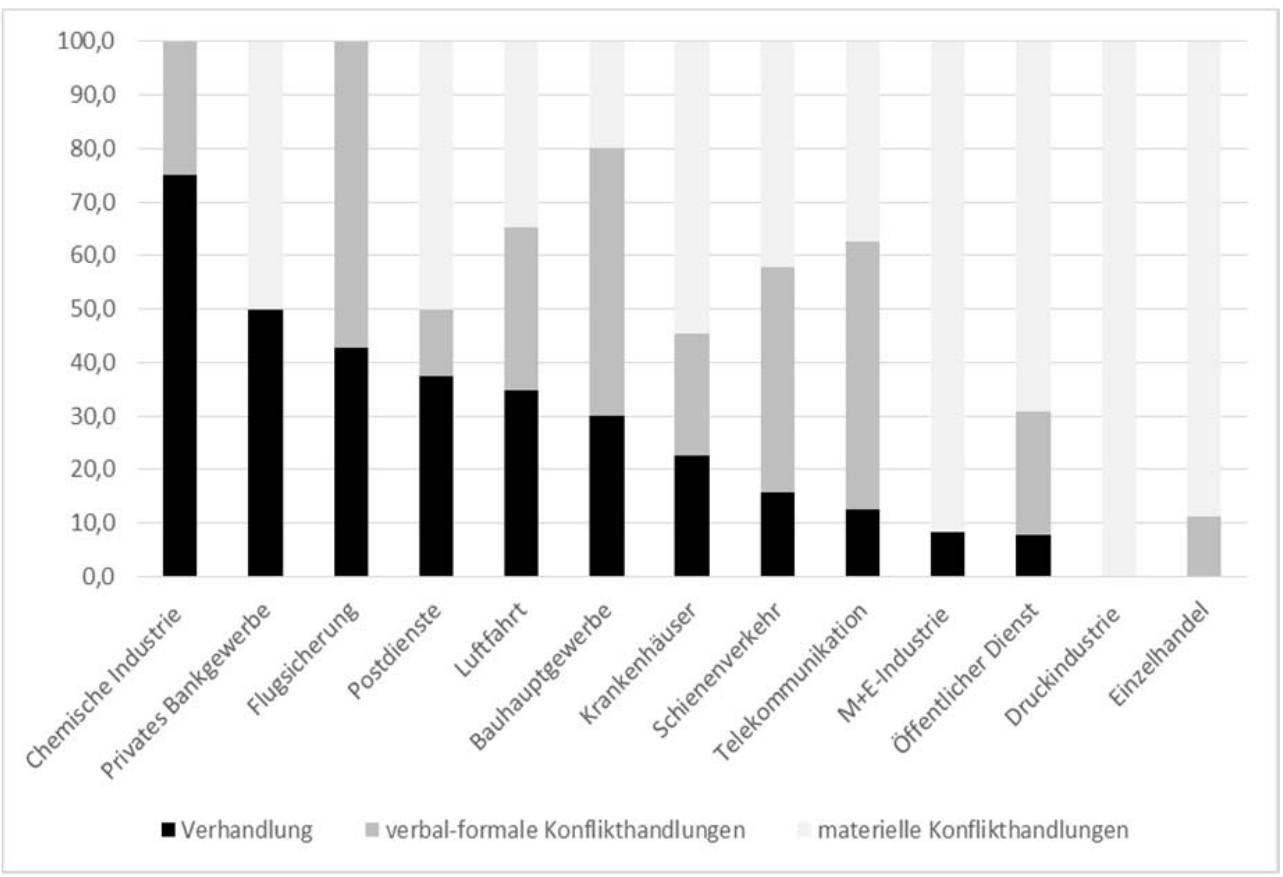

Abb. 3: Konfliktlösungskultur; Anteile der Konfliktlösungsansätze in Prozent

Auf der Basis dieser unterschiedlichen Anteile lassen sich vier Konfliktlösungskulturen voneinander unterscheiden. In der Chemischen Industrie wurden im Untersuchungszeitraum neun der zwölf Tarifrunden (75 Prozent) konfliktfrei - also nur durch Verhandlungen - gelöst. Hier dominierte also ein verhandlungsorientierter Ansatz. Daneben lassen sich drei Branchen identifizieren, in denen Konflikte zwar häufiger eskalierten, die Tarifparteien aber selten über verbale und formale Eskalationsstufen hinausgingen. Ein solcher verbalformaler Konfliktlösungsansatz lässt sich neben dem oben bereits erwähnten Bauhauptgewerbe auch in der Telekommunikation und in der Flugsicherung beobachten. In diesen Branchen lag der Anteil der Konflikte, die bis zu verbal-formalen Konflikthandlungen eskalierten, zwischen 50 und 57 Prozent. Materielle Konflikthandlungen spielten in der Flugsicherung gar keine Rolle, im Bauhauptgewerbe erreichten sie einen Anteil von lediglich 20 Prozent und in der Telekommunikation 37,5 Prozent.

In der materiell-konfliktorientierten Gruppe eskalierten Konflikte regelmäßig bis zum Warnstreik oder Streik. Ein solcher materieller Konfliktlösungsansatz war in der Druckindustrie seit 2002 sogar für jeden der sieben Tarifkonflikte charakteristisch. Auch in der Metall- und Elektro-Industrie (Anteil von 92 Prozent), im Einzelhandel (89 Prozent) sowie im Öffentlichen Dienst (69 Prozent) dominierte dieser Ansatz sehr deutlich. In Krankenhäusern und im Postdienst eskalierte immerhin noch jeder zweite Konflikt bis zu materiellen Konflikthandlungen.

In den drei übrigen Branchen Privates Bankgewerbe, Luftfahrt und Schienenverkehr dominierte keine der drei Strategien. Hier liegt eine heterogene Konfliktlösungskultur vor. 
In der Luftfahrt und im Schienenverkehr könnte dies daran liegen, dass mehrere Gewerkschaften verhandeln und sich dabei bestimmte Konfliktlösungsmuster überlagern. Dies wird durch eine Auswertung nach einzelnen Berufsgruppen aber nicht bestätigt. Die Konfliktlösungsmuster der dort vertretenen Gewerkschaften waren ebenso heterogen wie in der aggregierten Branchenbetrachtung. ${ }^{10}$ Eine Besonderheit lässt sich für das Private Bankgewerbe beobachten. Dort gab es jeweils zwei Strategien, die einen Anteil von genau 50 Prozent erreichen (Verhandlung oder materielle Konflikthandlungen). Hier liegt eine hybride Konfliktlösungskultur vor.

\section{Diskussion und Schlussfolgerungen}

Mit den neuen Vergleichsmaßen „maximale Eskalationsstufe“ und „Konfliktintensität“ wird im Rahmen dieses Beitrags der Versuch unternommen, Tarifverhandlungen genauer auf ihr Eskalationspotenzial hin zu überprüfen als es im Rahmen der üblichen Arbeitskampfindikatoren geschieht. Das Messen von Eskalationsstufen, die dem Arbeitskampf vorgelagert sind, bietet darüber hinaus die Möglichkeit, unterschiedliche Konfliktlösungskulturen $\mathrm{zu}$ identifizieren. Mit diesem methodischen und empirischen Vorgehen lässt sich nicht nur ein differenzierter Blick in die Tarifverhandlungsstrukturen einzelner Branchen werfen. Es bietet zugleich die Möglichkeit, den Begriff der „Konfliktpartnerschaft“ (Müller-Jentsch 1999; Müller-Jentsch 2013) empirisch auszuleuchten.

Der empirische Befund bestätigt, dass der Begriff der „Konfliktpartnerschaft“ nach wie vor eine passende Beschreibung des Verhältnisses zwischen Gewerkschaften und Arbeitgebern ist. Dass Streeck (2016) den Begriff „Konfliktpartnerschaft“ für überholt bewertet, wird durch den vorliegenden empirischen Befund nicht gestützt. In zwei der drei betrachteten Exportindustrien (Druckindustrie sowie Metall- und Elektro-Industrie) dominiert eindeutig ein materieller Konfliktlösungsansatz. Das Bild für die zehn untersuchten Dienstleistungsbranchen ist sehr gemischt. Es gibt Dienstleistungsbranchen, die nicht nur vergleichsweise konfliktarm verhandeln (Flugsicherung, Privates Bankgewerbe), sondern bei der Konfliktlösung neben Verhandlungen auch eher auf verbal-formale als auf materielle Konflikthandlungen setzen. Nur in drei der 13 untersuchten Branchen lassen sich keine eindeutigen Konfliktlösungsmuster identifizieren. Jede Tarifrunde verläuft anders, ein antizipierbares ,Ablaufritual“" stellt sich dadurch nicht ein.

Für Streecks (2016, S. 59) These vom „Konflikt ohne Partnerschaft“ spricht, dass Verhandlungen von Berufsgruppengewerkschaften besonders wenig vorsehbar sind. Dies hängt maßgeblich mit dem Wandel der Tarifverhandlungen zu Spartentarifverträgen zusammen, der bei den Berufsgruppengewerkschaften im Rahmen konfliktintensiver „Statuskonflikte“ ausgetragen wurde (Lesch \& Hellmich 2014, S. 367 ff.). Da sich die Arbeitgeber weigerten, die über Jahrzehnte praktizierte Tarifeinheit zugunsten einer Tarifpluralität aufzugeben, sahen sich die Berufsgruppengewerkschaften dazu genötigt, ihre verfassungsrechtlich geschützte Koalitionsfreiheit zu erzwingen. Tarifrunden, in denen solche Statusprobleme nicht auftraten, liefen in der Vergangenheit nicht erkennbar konfliktreicher ab als die von 
Branchengewerkschaften. Insofern scheint das Phänomen „Konflikt ohne Partnerschaft“ eher eine Momentaufnahme zu sein. In dem Maße, wie sich die derzeit agierenden fünf Spartengewerkschaften etabliert haben und das neue Tarifeinheitsgesetz einer weiteren Fragmentierung der Gewerkschaften vorbeugt, dürfte sich auch zwischen Arbeitgebern und Berufsgruppengewerkschaften eine Kultur der „Konfliktpartnerschaft“ etablieren. Zu beachten ist dabei allerdings, dass die Schnittmenge gemeinsamer Ziele bei Spartentarifverträgen geringer als bei Branchentarifverträgen sein dürfte, da Berufsgruppengewerkschaften spezifischere Arbeitnehmerinteressen vertreten als Branchengewerkschaften und daher auch weniger externe Effekte internalisieren.

Ein weiterer empirischer Befund ist, dass das Modell der „Sozialpartnerschaft“, in der es nur noch „gemeinsame Interessen und keine Konflikte mehr gibt“" (Streeck, 2016, S. 49) zumindest in der Chemischen Industrie gelebte Wirklichkeit ist. Ob die IG Bergbau, Energie, Chemie (IG BCE) dabei als „Juniorpartner“ auftritt, der „durch die wirtschaftlichen Verhältnisse zur Vernunft gezwungen wird“" (Streeck, 2016, S. 58), wäre zu diskutieren. Fest steht indes, dass der materiell-konfliktorientierte Lösungsansatz der IG Metall keine höheren Tariflohnsteigerungen hervorbringt als der verhandlungsorientierte Ansatz der IG BCE. Der Versuch, die Konfliktlösungskulturen in verschiedenen Branchen genauer zu untersuchen, ermöglicht es jedenfalls, die Oberbegriffe „Konfliktpartnerschaft“ und „Sozialpartnerschaft" auf einer anderen verbalen Ebene miteinander zu integrieren. Danach steht „Sozialpartnerschaft“ für einen verhandlungsorientierten Konfliktlösungsansatz und „Konfliktpartnerschaft" für verbal-formale oder materielle Konfliktlösungsansätze.

Eine offene Flanke der Bewertung von Konflikthandlungen besteht darin, dass weder die durch Streikdrohungen, Streikankündigungen, Warnstreiks oder Streiks verursachten Drittwirkungen noch die gesamtwirtschaftlichen Streikschäden berücksichtigt werden. Im Verkehrssektor führt schon eine Streikankündigung zu einem wirtschaftlichen Schaden. Sie wirkt dort anders als in einer Behörde oder in einem Industriezweig. Und auch die einzelnen Warnstreiks oder Streiks lassen sich im Hinblick auf die verursachten wirtschaftlichen Schäden selten über einen Kamm scheren. Hier stößt die Messung von Konfliktintensität auf dasselbe Problem wie die herkömmlichen Arbeitskampfindikatoren. Auch durch Streik und Aussperrung verlorene Ausfalltage sagen noch nichts über die dadurch verursachten wirtschaftlichen Schäden aus.

Eine weitere Grenze erfährt das Konzept der Eskalationsmessung dadurch, dass keine Zuordnung darüber möglich ist, welche Tarifpartei für die durchlaufenen Eskalationsstufen verantwortlich ist. Dieser Kritikpunkt trifft aber vor allem Auswertungen, die das Verhalten einzelner Gewerkschaften miteinander vergleichen (Lesch 2013a; Lesch \& Hellmich, 2014). Im Rahmen dieser Untersuchung wurde deshalb ganz bewusst auf eine Zurechnung auf einzelne Tarifakteure verzichtet und ein Branchenvergleich vorgenommen. Dabei wird die Frage, wieviel „Schuld“ eine einzelne Tarifpartei an einer Konflikteskalation hat, ausgeklammert.

Natürlich wirft auch die Anordnung der Konflikthandlungen im Rahmen einer Eskalationspyramide Fragen auf. Die Verortung der Schlichtung wurde in Kapitel 2.3 bereits ausführlich diskutiert. Ein weiteres Problem betrifft die Druckmittel der Arbeitgeber. Auf einen Streik mit einer Aussperrung zu antworten, ist eher selten geworden. Relevanter ist, dass Arbeitgeber mit der Möglichkeit einer Produktionsverlagerung über ein wirksameres 
Druckmittel verfügen, um die Gewerkschaften zu disziplinieren und konfliktreiche Auseinandersetzungen auch zu sanktionieren. Gerade die erst durch die Globalisierung möglich gewordene Produktionsverlagerung stellt für Streeck (2016) eine entscheidende strukturelle Machtverschiebung dar, die die Gewerkschaften in die erwähnte Rolle eines ,Juniorpartners“ drängt. Allerdings hat Lesch (2013b, S. 27 ff.) im Rahmen eines einfachen NashVerhandlungsspiels gezeigt, dass die Verbesserung des Arbeitgeberdrohpunkts durch eine zusätzliche Ausweichoption nicht die Machtrelation zwischen den Tarifparteien verändert, sondern lediglich die Verteilungsrelation. Durch eine Verlagerungsoption wird schlichtweg der lohnpolitische Verteilungsspielraum geringer. Sie verhindert aber nicht, dass eine Gewerkschaft die (dann freilich kleinere) Produktion durch Streiks vollständig lahmlegen kann. Die Konflikthandlung „Streik“ bleibt der Gewerkschaft damit unbenommen.

Inwieweit sie bei der Abwägung darüber, ob sie eine bestimmte Eskalationsstufe wählt, auf „ökonomische Sachzwänge“ Rücksicht nimmt, kann eine Konfliktmessung nicht abbilden. Genauso wenig kann sie abbilden, dass Arbeitgeber einem Tarifkompromiss zustimmen, weil sie eine Streikdrohung fürchten. Messbar sind nur Konflikthandlungen, nicht die Motive, die hinter den Konflikthandlungen stehen. Die konfliktsoziologisch relevante Fragestellung des Ansatzes lautet: Welche Austragungsformen lassen sich bei den untersuchten Konflikten unterscheiden? ${ }^{11}$ Ungeachtet dessen, was konfliktsoziologisch untersucht werden soll, scheitert eine Berücksichtigung von Produktionsverlagerungen als Konflikthandlung schon praktisch daran, dass sich Produktionsverlagerungen selten auf einen konkreten Arbeitskampf zurückführen lassen, geschweige denn, einzelnen Tarifkonflikten eindeutig zugeordnet werden können.

Die durch die empirischen Befunde angestoßenen und offenen Forschungsfragen lauten: Warum lassen sich für einzelne Branchen verschiedene Konfliktlösungskulturen identifizieren? Gibt es Pfadabhängigkeiten, sind es exogene Einflüsse oder sogar endogene? Die durch die Globalisierung ermöglichte Produktionsverlagerung könnte durchaus einen solchen exogenen Einfluss darstellen, der zu einer Verhaltensänderung und damit zu einer neuen Konfliktlösungskultur führt. Um dies empirisch zu überprüfen, müsste der Untersuchungszeitraum in die 1980er Jahre hinein ausgedehnt werden. Genauso können aber auch endogene Prozesse prägend wirken. Die „Sozialpartnerschaft“ in der Chemischen Industrie wird oft auf den gescheiterten Streik zu Beginn der 1970er Jahre zurückgeführt. Genauso entscheidend könnten aber auch die handelnden Personen sein. Hier eröffnet sich demnach ein weites Forschungsfeld für Experteninterviews und Fallstudien.

\section{Literatur}

Aligisakis, M. (1997). Labour Disputes in Western Europe: Typology and Tendencies. In: International Labour Review, 136(1), 73-94.

Ashenfelter, J. \& Johnson, G. E. (1969). Bargaining Theory, Trade Unions and Industrial Strike Activity. American Economic Review, 59(1), 35-49.

Berg, P., Platow, H., Schoof, C. \& Unterhinninghofen, H. (2010). Tarifvertragsgesetz und Arbeitskampfrecht. Kompaktkommentar. Frankfurt am Main.

11 Diese Frage ist eine der konfliktsoziologisch zentralen Fragestellungen (Bonacker, 2008, S. 16). Zur Frage, was Gegenstände von Tarifkonflikten sein können, siehe Lesch \& Hellmich (2014). Dort wird zwischen Status- und Tarifkonflikten unterschieden (konfliktsoziologisch: zwischen teilbaren und unteilbaren Konflikten). 
Bonacker, T. (2008). Sozialwissenschaftliche Konflikttheorien - Einleitung und Überblick. In: T. Bonacker (Hrsg.): Sozialwissenschaftliche Konflikttheorien. Eine Einführung. 4. Auflage. Wiesbaden, 9-29.

Bordogna, L., Cella, G. P. (2002). Decline or transformation? Change in industrial conflict and its chal-lenge. In: Transfer, 8(4), 585-607. https:/doi.org/10.1177/102425890200800401

Carley, M. (2013). Developments in industrial action 2005-2009, http://www.eurofound.europa.eu/observatories/eurwork/comparative-information/developmentsin-industrial-action-2005-2009 [19.1.2016].

Crampton, P., Gunderson, M. \& Tracy, J. (1999). The Effect of Collective Bargaining Legislation on Strikes and Wages. In: Review of Economic and Statistics, 81(3), 475-487. https:/doi.org/10.1162/003465399558238

Däubler, W. (2011). Arbeitskampfrecht. Handbuch für die Rechtspraxis. Baden-Baden.

Gärtner, M. (1989): Arbeitskonflikte in der Bundesrepublik Deutschland - Eine empirische Untersuchung ihrer makroökonomischen Ursachen und Konsequenzen, Berlin.

Hicks, J. R. (1963). The Theory of Wages. London.

https:/doi.org/10.1007/978-1-349-00189-7

Keller, B. (1975). Determinanten des Schlichtungsprozesses: Konfliktmanagement durch Intervention Dritter. In: Kyklos, 28(1), 117-142. https:/doi.org/10.1111/j.1467-6435.1975.tb01937.x

Keller, B. (1985). Schlichtung als autonomes Regelungsverfahren der Tarifparteien. In: Endruweit G. et al.: Handbuch der Arbeitsbeziehungen, 119-130, Berlin.

Külp, B. (1969). Streik und Streikdrohung. Ihre Rolle in der Volkswirtschaft und im Sozialprozess, Berlin.

Külp, B., Keller B., Wiedemann K. \& Wolters, R. (1972). Der Einfluss von Schlichtungsformen auf Verlauf und Ergebnis von Tarif- und Schlichtungsverhandlungen, Berlin.

Kunz, V. (2008). Die Konflikttheorie des Rational Choice-Theorie. In: T. Bonacker (Hrsg.): Sozialwissenschaftliche Konflikttheorien. Eine Einführung. 4. Auflage. Wiesbaden: 461-484.

Lesch, H. (2002). Streik und Arbeitskampfregeln im internationalen Vergleich. In: IW-Trends 29(2). 5-17.

Lesch, H. (2013a). Die Konfliktintensität von Tarifverhandlungen. In: IW-Trends, 40(3). 63-79.

Lesch, H. (2013b). Ökonomik des Arbeitskampfrechts. In: IW-Analysen, Nr. 86, Köln.

Lesch, H. (2016). Flächentarifvertrag und Tarifpartnerschaft in Deutschland: Ein historischer Rückblick. In: IW-Analysen, Nr. 107, Köln.

Lesch, H. \& Hellmich, P. (2014). Das Tarifeinheitsgesetz: Juristischer Balanceakt mit ökonomischer Wirkung. In: List Forum für Wirtschafts- und Finanzpolitik 40(4). 352-381.

Müller-Jentsch, W. (1999). Konfliktpartnerschaft: Akteure und Institutionen der industriellen Beziehungen, 3. Auflage. München.

Müller-Jentsch, W. (2013). Gewerkschaften und Korporatismus: Vom Klassenkampf zur Konfliktpartnerschaft. In: Führer, K. C./Mittag, J./Schildt. A./Tenfelde, K./Jäger, W. (Hrsg.): Revolution und Arbeiterbewegung in Deutschland 1918-1920. Essen: 81:96.

Posner, R. A. (2007). Economic Analysis of Law, Austin.

Rieble, V. (2005). Modernisierung des Arbeitskampfrechts zu einem Tarifverhandlungsrecht. In: Zeitschrift für Arbeitsmarktforschung, 38(2/3): 218-229.

Ross, A.M. \& Hartmann, P.T. (1960). Changing Patterns of Industrial Conflict. New York.

Schnabel, C. (1989). Zur ökonomischen Analyse der Gewerkschaften in der Bundesrepublik Deutschland, Frankfurt a. M.

Siebert, W.S., \& Addison, J.T. (1981). Are Strikes Accidental?. In: Economic Journal, 91(362): 389-404.

Streeck, W. (2016). Von Konflikt ohne Partnerschaft zu Partnerschaft ohne Konflikt. In: Industrielle Beziehungen 23 (1): 47-60. doi 10.1688/IndB-2016-01-Streeck. 
Vandaele, K. (2011). Sustaining or abandoning "social peace"? Strike developments and trends in Europe since the 1990s. In: ETUI Working Paper Series, No. 2011.05, Brüssel.

Zerche, J. (1970). Lohnfindung durch Tarifverhandlungen - Neue Ansätze in der Collective Bargaining-Forschung, Tübingen.

Zerche, J. (1979). Arbeitsökonomik, Berlin. 


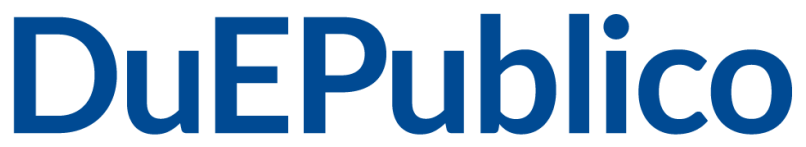

Duisburg-Essen Publications online

\section{DESISB B R R}

offen im Denken

$\mathbf{U b} \mid \begin{aligned} & \text { universitäts } \\ & \text { bibliothek }\end{aligned}$

Dieser Text wird über DuEPublico, dem Dokumenten- und Publikationsserver der Universität Duisburg-Essen, zur Verfügung gestellt. Die hier veröffentlichte Version der EPublikation kann von einer eventuell ebenfalls veröffentlichten Verlagsversion abweichen.

DOI: $\quad$ 10.3224/indbez.v24i1.03

URN: urn:nbn:de:hbz:464-20200717-112810-7 